Rare disease: Primary leiomyoma of the liver: accurate preoperative diagnosis on liver... Page 1 of 9

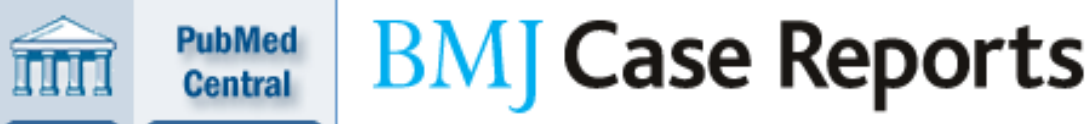

Search Journal List Current TOC | Instructions for authors

BMJ Case Rep. 2009; 2009: bcr09.2008.0898

Published online 2009 March 24. doi: 10.1136/bcr.09.2008.0898

PMCID: PMC3027874

Copyright 2009 BMJ Publishing Group Ltd

Rare disease

\section{Primary leiomyoma of the liver: accurate preoperative diagnosis on liver biopsy}

\author{
Helena T Sousa, ${ }^{1}$ Francisco Portela, ${ }^{1}$ Luis Semedo, ${ }^{2}$ Emanuel Furtado, ${ }^{3}$ Carol Marinho, ${ }^{4}$ Maria A \\ Cipriano, ${ }^{4}$ and Maximino C Leitão ${ }^{1}$
}

${ }^{1}$ Department of Gastroenterology, Coimbra University Hospital, Praceta Mota Pinto, Coimbra 3000-075, Portugal

${ }^{2}$ Department of Radiology, Coimbra University Hospital, Praceta Mota Pinto, Coimbra 3000-075, Portugal

${ }^{3}$ Department of Surgery II, Coimbra University Hospital, Praceta Mota Pinto, Coimbra 3000-075, Portugal

${ }^{4}$ Department of Pathology, Coimbra University Hospital, Praceta Mota Pinto, Coimbra 3000-075, Portugal

H T Sousa, Email: helenatsousa@gmail.com

\begin{abstract}
Primary leiomyoma of the liver is an exceptionally rare tumour in non-immunocompromised patients. Preoperative diagnosis of the lesion is difficult as complete imaging of this type of lesion is scarcely defined and preoperative biopsy was not the practice in previously reported cases. We report a voluminous primary leiomyoma of the liver occurring in a healthy middle-aged woman where a preoperative diagnosis was accurately achieved on biopsy. Because of its size, surgery was undertaken for exclusion of malignancy. A 16-month uneventful follow-up has been completed. We discuss the advantage of a preoperative diagnosis and propose that an imaging-guided liver biopsy should be undertaken, provided malignancy features are absent. This could prevent liver surgery merely for diagnostic purposes. Finally, we report imaging features that have not been previously described, namely on magnetic resonance imaging, which may provide an insight about the nature of this particular lesion and, advantageously, contribute toward a non-invasive diagnosis.
\end{abstract}

\section{BACKGROUND}

We report a case of an incidental focal hepatic lesion detected in the course of routine investigation of a previously healthy middle-age patient, similarly to so many patients in our practice. However, this focal lesion was an exceptionally rare liver tumour that led to liver surgery, despite a histological preoperative diagnosis had been achieved. This was undertaken mostly because primary leiomyomas of the liver are poorly understood and scarcely described lesions. Yet, although rare, these lesions seem to have an excellent prognosis and no associated malignancy has been described so far. Thus, we propose a preoperative diagnosis should be warranted as it may prevent diagnostic liver surgery. We demonstrate that a histological preoperative diagnosis is possible and we further describe detailed and new imaging features, which might contribute to the definition of a radiological pattern.

\section{CASE PRESENTATION}

A 61-year-old woman consulted her family physician for dyspepsia continuing for over 6 months. Her medical history included transvaginal resection of two simple endometrial polyps 15 months earlier. She had her menopause at age 56 , denied risk behaviours and alcohol, tobacco or drug abuse and had no history of liver disease or blood transfusions. Her great grandfather had died of colorectal cancer in his eighties and her father had died of prostate cancer at the age of 88 . Physical examination was unremarkable with no discomfort or mass found on abdominal examination.

\section{INVESTIGATIONS}

Haematological and biochemical parameters were within normal ranges. Tumour markers a-fetoprotein, carcinoembryonic antigen and carbohydrate antigens 19.9, 125 and 15.3 were also normal. An upper 
Rare disease: Primary leiomyoma of the liver: accurate preoperative diagnosis on liver... Page 2 of 9

gastrointestinal endoscopy and left colonoscopy had been performed 5 months before, with no lesions being found. Abdominal ultrasound (US) showed heterogeneity of segments II and III of the liver related to several confluent hypoechoic nodules, the largest measuring $3.4 \mathrm{~cm}$. Pelvic US was normal. Computed tomography showed a slightly hypodense $7.8 \mathrm{~cm}$ mass on the lateral segments of the left hepatic lobe with strong arterial phase enhancement and remaining hyperdense in subsequent enhancement phases, where it simulated a polylobulated form (fig 1 ). A $1 \mathrm{~cm}$ hypodense nodule on segment IV suggestive of a biliary cyst was also noted. No other abnormalities were found in any other part of the body, except for a $1 \mathrm{~cm}$ nodule on the left adrenal gland, which behaved like an adenoma. In the T2-weighted fat-suppressed sequence, magnetic resonance imaging (MRI) showed a low-signal intensity mass in the left liver lobe, which was also hypointense in the T1-weighted image. The gadolinium-enhanced dynamic study showed a marked, progressive enhancement throughout the liver enhancement phases (fig 2). As imaging was inconclusive, she was referred to our hospital for further evaluation. We performed a US-guided fine needle aspiration (FNA) and an 18G tru-cut biopsy of the tumour for cytological and histological examination, respectively. At this time we noticed a somewhat surprising hardness of the lesion. Cytological examination was inconclusive as the material was scarce and included only a small group of normal hepatocytes. Microscopic examination of the 13 $\mathrm{mm}$ biopsy cylinder revealed hypocellular material, composed of fusocellular connective tissue with a hyaline collagen matrix and numerous vascular structures and no inflammatory component. Nuclear hyperchromatism and pleomorphism were low and no mitotic figures were found. There was no hepatic or epithelial tissue.

Immunohistochemistry (IH) showed strong and diffuse reactivity for smooth muscle actin (AML) and HHF35 (striated and smooth muscle actin) but not for S-100, CD117 (c-kit), HMB45 and CD34. They were also negative for bcl2 and CD99. Vascular endothelia were only stained for CD34.

\section{TREATMENT}

Despite the apparently benign histological features of the lesion, its atypical enhancement on imaging studies and large size could not exclude malignant potential. This and the technical feasibility of the appropriate surgery were explained to the patient and she underwent a left hepatectomy and left adrenalectomy. Manual palpation of the small bowel was performed during surgery with no nodule of the wall noted.

\section{OUTCOME AND FOLLOW-UP}

Macroscopically the liver tumour was located subcapsularly, $1.5 \mathrm{~cm}$ from the surgical margin. It was an ovoid mass, measuring $90 \times 95 \times 60 \mathrm{~mm}$, consisting of pink-whitish hard-elastic fasciculate tissue with boundaries clearly demarcated from the surrounding liver tissue ( $\underline{\mathrm{fig}} 3$ ). Microscopic examination confirmed the fusocellular nature of the tumour, composed of low-cellular density anastomosing fascicles of uniform spindle cells with no atypia or mitotic figures. It depicted a rich vascular network with occasional eccentric thickening of vascular walls, mixoid alterations and perivascular oedema. There were also extensive areas of hyalinisation. The growth pattern was expansive, there was no hepatic tissue inside the tumour and the surrounding hepatic parenchyma showed features of compression. IH confirmed the features observed in the previous biopsy (fig 4). The fusocellular population was strongly reactive for vimentin and $\mathrm{AML}$ and showed negativity for calponin, desmin, $\mathrm{CK} 35 \mathrm{BH} 11$ and oestrogen receptors. Very low $(<2 \%)$ nuclear staining for Ki67 confirmed the reduced rate of cellular proliferation. The adrenal gland examination revealed micronodular hyperplasia of the cortical layer. The postoperative period was uneventful. After surgery the patient underwent upper gastrointestinal endoscopy and total colonoscopy that did not show any submucosal lesions. Sixteen months after surgery she is doing well, although still complaining of occasional dyspepsia.

\section{DISCUSSION}

Incidental focal hepatic lesions are being increasingly detected in the course of routine or symptom-based investigation of patients. The very high diagnostic accuracy of liver imaging today relies on specific characteristics or dynamic patterns shown by most focal liver lesions. Rare lesions of the liver are still a challenge for the radiologist as their imaging behaviour is often variable and known patterns must be carefully looked for. On US, hepatic leiomyomas have been repeatedly described as hypoechoic lesions with variable degrees of heterogeneity. ${ }^{1-3}$ On computed tomography most authors reported hypodense lesions with strong enhancement in both arterial and portal 
phases with some authors also describing a peripheral rim enhancement. ${ }^{1,2}$ On angiography, marginal, ${ }^{1}$ diffuse ${ }^{3}$ or irregular ${ }^{2}$ hypervascularisation were variously reported. As for MRI, these lesions were hypointense in T1-weighted images, partially hyperintense in T2-weighted sequences ${ }^{1,2}$ and showed marked gadolinium enhancement in both early and equilibrium phases. ${ }^{2}$ Behaviour after administration of ferumoxides was variable. Our case confirmed the hypervascularity features reported on computed tomography and MRI ( fig 1B-D; fig 2C-E). In addition, the lesion presented hypointensity in the T2-weighted MRI images, which had not been previously described, and may be related to its dense fusocellular nature (fig 2A). In fact, both smooth and skeletal muscle possess low signal intensity on T2-weighted images, which results from the T2 shortening effects of intramuscular actin, myosin and collagen and decreased extracellular fluid compared with surrounding tissues. As a result, the low signal intensity on T2weighted images may provide an insight into the tissue components of this kind of focal liver lesions. Thus, although a typical pattern still cannot be defined for these lesions, it seems likely that hypervascular imaging features, related to the enlarged capillary vessels noted on histology, might be a characteristic of primary leiomyoma of the liver. In addition, it has been suggested that hypervascularity could favour leiomyoma rather than leiomyosarcoma. ${ }^{1}$ However, imaging alone cannot reliably differentiate between primary leiomyoma of the liver, hepatocellular adenoma or carcinoma, fibrolamellar liver carcinoma, peripheral cholangiocellular carcinoma, angiomyolipoma and, more commonly found, hypervascular liver metastasis. ${ }^{2}$ If not for all, then for most of these hypervascular focal lesions of the liver, the final diagnosis still depends on tissue examination.

Diagnosis of primary leiomyoma of the liver requires two criteria: the tumour should originate from the mesenchymal tissue of the liver, and there should be no primary tumour elsewhere in the body. ${ }^{1}$ In our case, cytological examination was inconclusive because of insufficient material. A recent series of spindle cell lesions of the liver evaluated by FNA reported the same difficulty in $10 \%$ of cases. ${ }^{4}$ Furthermore, while smooth muscle origin of leiomyosarcomas can be demonstrated by $\mathrm{IH}$ studies, this is not always possible with FNA material. ${ }^{4}$ Thus, FNA does not seem adequate for an accurate diagnosis of leiomyoma. Indeed, FNA is considered more suitable for differentiating a malignant from a benign liver lesion while biopsy is better for discriminating the type of benign tumour. ${ }^{5}$ However, an estimated risk of tumoral seeding of 1:10 000 for FNA and up to $2 \%$ for biopsy ${ }^{5}$ should be taken into account. In our case, imaging was inconclusive but there were no clinical or laboratory signs of malignancy and biopsy seemed to be an advantage. Indeed most of the $\mathrm{IH}$ features found in biopsy material were later confirmed in the resected specimen. Thus an accurate preoperative diagnosis of leiomyoma was achieved by means of tru-cut liver biopsy. To the best of our knowledge this is the first report of a preoperative diagnosis of a primary leimyoma of the liver. Recently, Beuzen et $a^{\beta}$ suggested that tumour biopsy is appropriate for asymptomatic lesions, while primary surgical resection should be performed when there are symptoms. This is questionable as it is known that the malignant potential of smooth muscle tumours increases with their diameter, and metastasis and recurrence of a tumour initially diagnosed as leiomyoma have been described. ${ }^{1}$ On the other hand, although still poorly understood due to their rarity, these lesions seem to have an excellent prognosis. Most of the cases described are in female patients in their fifties, found after investigation for mass-related abdominal signs and/or symptoms. ${ }^{1-3}$ All but one case had surgical resection and an unremarkable outcome. The non-operated case concerned a liver leiomyoma found incidentally during the autopsy of an 87-year-old woman who died from upper gastrointestinal bleeding. ${ }^{2}$ This preoperative diagnosis could allow a wait-and-see approach in selected casessmall or deeply located lesions and non-surgical patients, for example-instead of a universal surgical solution.

In our case, IH depicted intense and diffuse staining of spindle cells for striated and smooth muscle actin and vimentin (fig 4) but not for S-100, CD117 (c-kit), HMB45 and CD34. They were also negative for calponin, desmin, low-molecular-weight $\mathrm{CK}, 35 \mathrm{BH} 11, \mathrm{ER}, \mathrm{Bc} 22$ and $\mathrm{CD} 99$. A rich vascularisation was confirmed by CD34 endothelial staining. It seems that positive staining for smooth muscle actin could be a hallmark for this type of lesion because it was described in most reported cases involving detailed immunostaining studies. ${ }^{1-3}$ Staining for vimentin or desmin was sometimes observed. ${ }^{2,3}$ Caution is required when it comes to distinguishing between leiomyoma and gastrointestinal stromal tumour (GIST), which can be difficult even on histology. Although they also feature the interlacing proliferation of spindle-shaped cells, GISTs are almost always positive for c-kit, ${ }^{2}$ which is not found in digestive leiomyomas. ${ }^{3}$ Reactivity for CD34 can be found in about $70 \%$ of GISTs, ${ }^{3}$ in solitary fibrous tumours of the liver ${ }^{6}$ and occasionally in uterine but not in digestive leiomyomas. ${ }^{3}$ Staining for both c-kit and CD34 was not found in our case, thereby excluding GISTs and solitary fibrous tumours of the liver. An anecdotal case of a primary leiomyoma of the liver positive for c-kit and CD34 was reported by Beuzen et al, ${ }^{3}$ illustrating the still undefined 
Rare disease: Primary leiomyoma of the liver: accurate preoperative diagnosis on liver... Page 4 of 9

nature of these lesions. Hepatic angiomyolipoma was excluded due to negative reactivity for c-kit and HMB45. ${ }^{3}$ Finally, inflammatory myofibroblastic tumour was unlikely because no inflammatory component was observed.

Benignity of the lesion is suggested by the absence of nuclear atypia and mitotic figures on haematoxylin and eosin staining, and by a very low rate of cellular proliferation given by Ki67. However, as the natural course of this rare lesion has still not been defined, the only valid criteria of benignity so far should be no recurrence in the long term. ${ }^{3}$ Similarly, a primary origin can only be inferred by the impossibility of demonstrating synchronous or metachronous digestive or genital leiomyomas. Our patient has completed a 1.5-year follow-up with no other smooth muscle tumours identified. This reinforces the likelihood of both a benign nature and a primary origin of the tumour, but close clinical and imaging follow-up is warranted.

In conclusion, we report a rare case of a primary leiomyoma of the liver in an asymptomatic middle-aged woman. Its unique imaging features should be added to previously reported aspects. In the absence of features suggestive of malignancy we propose a preoperative diagnosis by means of an imaging-guided liver biopsy. This can be of advantage in the management of small lesions or whenever a surgical solution is not easily feasible.

\section{LEARNING POINTS}

- Primary leiomyoma of the liver is an exceptionally rare tumour in non-immunocompromised patients that seem to have excellent prognosis.

- Preoperative diagnosis should be warranted as it may prevent diagnostic liver surgery.

- Histological preoperative diagnosis is possible by means of imaging-guided tru-cut liver biopsy.

- Detailed, new imaging features are herein described. In particular, when a hypervascular focal liver lesion presents hypointensity on T2-weighted MR images, which may be related to its dense fusocellular nature, the diagnosis of leiomyoma should be taken into account.

\section{Footnotes}

Competing interests: none.

Patient consent: Patient/guardian consent was obtained for publication.

\section{REFERENCES}

1. Urizono Y, Ko S, Kanehiro H, et al. Primary leiomyoma of the liver: report of a case. Surg Today 2006; 36:629-32. [PubMed: 16794799]

2. Kanazawa N, Izumi N, Tsuchiya K, et al. A case of primary leiomyoma of the liver in a patient without evidence of immunosuppression. Hepatol Res 2002; 24: 80-8. [PubMed: 12243795]

3. Beuzen F, Roudie J, Moali I, et al. Léiomyome du foie: une tumeur benigne exceptionelle. Gastroenterol Clin Biol 2004; 28: 1169-72. [PubMed: 15657544]

4. Guy CD, Yuan S, Ballo MS. Spindle-cell lesions of the liver: diagnosis by fine-needle aspiration biopsy. Diagn Cytopathol 2001; 25: 94-100. [PubMed: 11477711]

5. Scoazec JY, Labadie M, Dumortier J, et al. Diagnostic des nodules hépatiques: techniques, démarche et principaux problèmes pratiques. Gastroenterol Clin Biol 2000; 24: 1095-103. [PubMed: 11139677]

6. Saint-Marc O, Pozzo A, Causse X, et al. Tumeur fibreuse solitaire du foie: caractéristiques cliniques, radiologiques et anatomo-pathologiques. Gastroenterol Clin Biol 2002; 26: 171-3. [PubMed: 11938069] 
Rare disease: Primary leiomyoma of the liver: accurate preoperative diagnosis on liver... Page 5 of 9

\section{Figures and Tables}
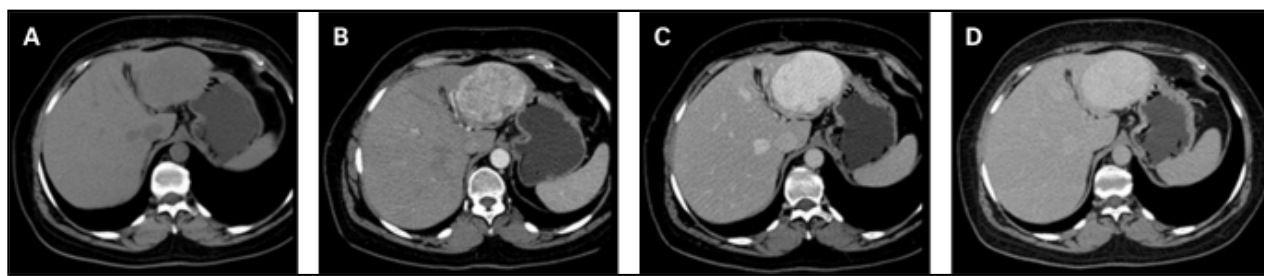

Figure 1

Computed tomography: (A) Unenhanced examination shows a slightly hypoattenuating left lobe mass. (B-D) In the dynamic study the lesion demonstrates a marked, progressive and homogeneous enhancement throughout the liver enhancement phases. 
Rare disease: Primary leiomyoma of the liver: accurate preoperative diagnosis on liver... Page 6 of 9
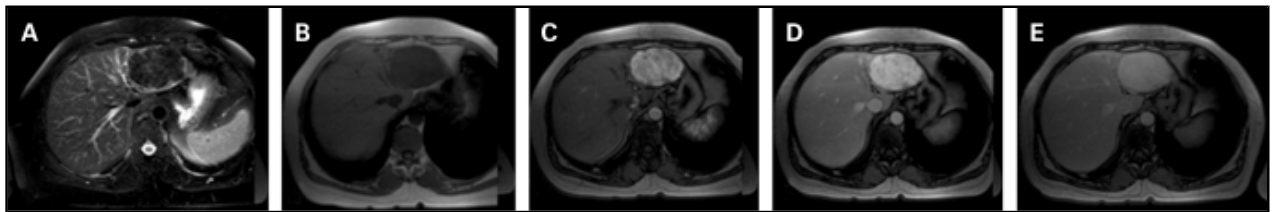

\section{Figure 2}

Magnetic resonance imaging: (A) T2-weighted fat-suppressed sequence depicts a low signal intensity mass in the left liver lobe, which is also hypointense in the T1-weighted image (B). In the dynamic study (C-E) it reveals a marked, progressive enhancement throughout the liver enhancement phases. 
Rare disease: Primary leiomyoma of the liver: accurate preoperative diagnosis on liver... Page 7 of 9

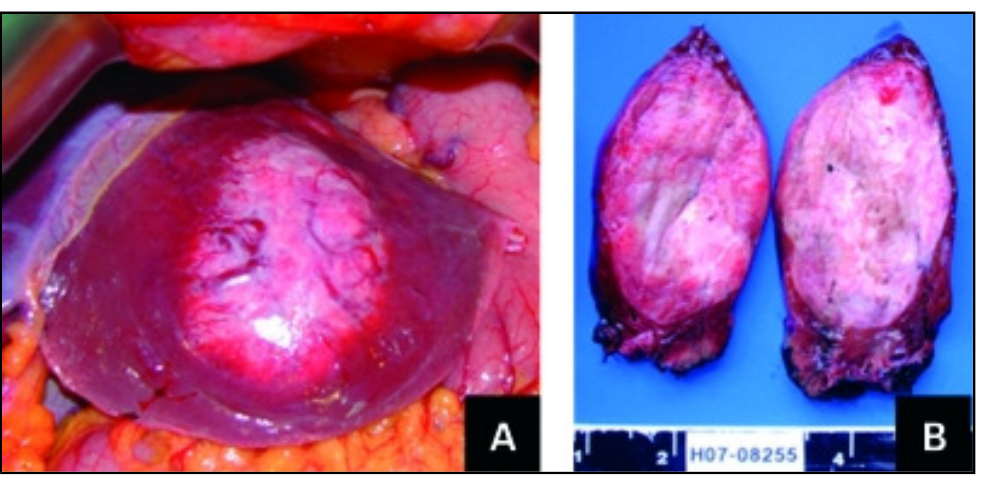

Figure 3

Primary leiomyoma of the liver. (A) In situ. Hepatic left lobe in view. (B) Macroscopic examination (in section). 
Rare disease: Primary leiomyoma of the liver: accurate preoperative diagnosis on liver... Page 8 of 9

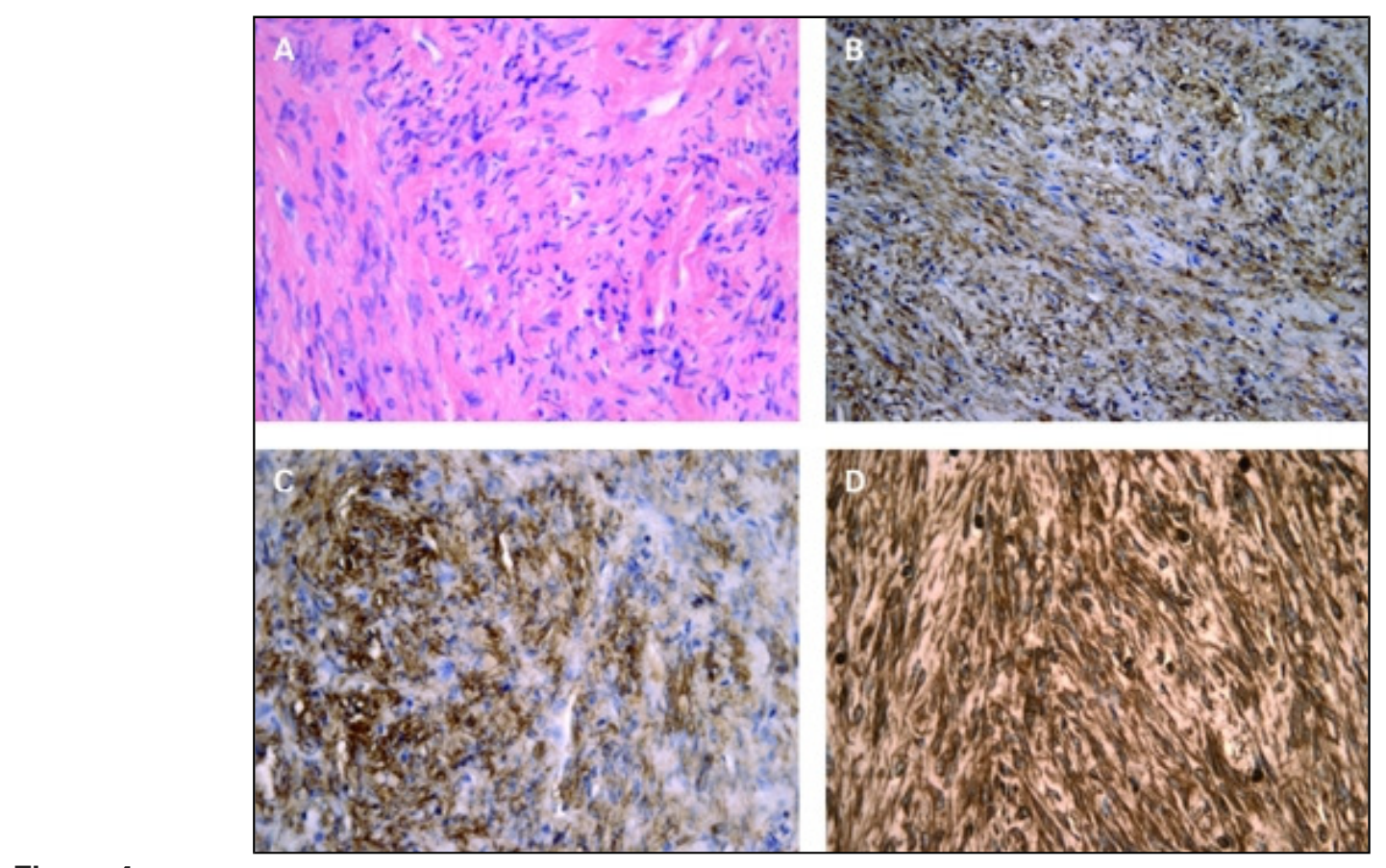

\section{Figure 4}

Primary leiomyoma of the liver staining characterisation. (A) Haematoxylin-eosin, $\times 400$. (B) AML, $\times 200$. (C) HHF35, $\times 400$. (D) Vimentin, $\times 400$.

Articles from BMJ Case Reports are provided here courtesy of BMJ Group 
Rare disease: Primary leiomyoma of the liver: accurate preoperative diagnosis on liver... Page 9 of 9 